\title{
Consensus Recommendations to NCCIH from Research Faculty in a Transdisciplinary Academic Consortium for Complementary and Integrative Health and Medicine
}

\author{
Martha Brown Menard, PhD, LMT, John Weeks, ${ }^{1}$ Belinda Anderson, PhD, LAc, ${ }^{3}$ \\ William Meeker, DC, MPH, ${ }^{4}$ Carlo Calabrese, ND, $\mathrm{MPH}^{5}{ }^{5}$ \\ David O'Bryon, JD, CAE, ${ }^{6}$ and Greg D. Cramer, DC, $\mathrm{PhD}^{7}$
}

\begin{abstract}
Background: This commentary presents the most impactful, shared priorities for research investment across the licensed complementary and integrative health $(\mathrm{CIH})$ disciplines according to the Academic Consortium for Complementary and Alternative Health Care (ACCAHC). These are (1) research on whole disciplines; (2) costs; and (3) building capacity within the disciplines' universities, colleges, and programs. The issue of research capacity is emphasized.

Discussion: ACCAHC urges expansion of investment in the development of researchers who are graduates of $\mathrm{CIH}$ programs, particularly those with a continued association with accredited CIH schools. To increase capacity of $\mathrm{CIH}$ discipline researchers, we recommend National Center for Complementary and Integrative Health (NCCIH) to (1) continue and expand R25 grants for education in evidence-based healthcare and evidence-informed practice at $\mathrm{CIH}$ schools; (2) work to limit researcher attrition from $\mathrm{CIH}$ institutions by supporting career development grants for clinicians from licensed $\mathrm{CIH}$ fields who are affiliated with and dedicated to continuing to work in accredited CIH schools; (3) fund additional stand-alone grants to $\mathrm{CIH}$ institutions that already have a strong research foundation, and collaborate with appropriate National Institutes of Health (NIH) institutes and centers to create infrastructure in these institutions; (4) stimulate higher percentages of grants to conventional centers to require or strongly encourage partnership with CIH institutions or $\mathrm{CIH}$ researchers based at $\mathrm{CIH}$ institutions, or give priority to those that do; (5) fund research conferences, workshops, and symposia developed through accredited CIH schools, including those that explore best methods for studying the impact of whole disciplines; and (6) following the present NIH policy of giving priority to new researchers, we urge $\mathrm{NCCIH}$ to give a marginal benefit to grant applications from CIH clinician-researchers at $\mathrm{CIH}$ academic/research institutions, to acknowledge that $\mathrm{CIH}$ concepts require specialized expertise to translate to conventional perspectives.
\end{abstract}

Summary: We commend NCCIH for its previous efforts to support high-quality research in the CIH disciplines. As NCCIH develops its 2016-2020 strategic plan, these recommendations to prioritize research based on whole disciplines, encourage collection of outcome data related to costs, and further support capacity-building within $\mathrm{CIH}$ institutions remain relevant and are a strategic use of funds that can benefit the nation's health.

\footnotetext{
${ }^{1}$ Crocker Institute, Kiawah Island, SC.

${ }^{2}$ Academic Consortium for Complementary and Alternative Health Care, Seattle, WA

${ }^{3}$ Pacific College of Oriental Medicine, New York, NY.

${ }^{4}$ Palmer College of Chiropractic, San Jose, CA.

${ }^{5}$ Center for Natural Medicine, Portland, OR.

${ }^{6}$ Association of Chiropractic Colleges, Bethesda, MD.

${ }^{7}$ National University of Health Sciences, Lombard, IL.
}

(C) Menard et al. 2015; Published by Mary Ann Liebert, Inc. This Open Access article is distributed under the terms of the Creative Commons Attribution Noncommercial License (http://creativecommons.org/licenses/by-nc/4.0/) which permits any noncommercial use, distribution, and reproduction in any medium, provided the original author(s) and the source are credited. 
Background of the Academic Consortium for Complementary and Alternative Health Care

$\mathbf{T}$ He Academic Consortium for Complementary and Alternative Health Care (ACCAHC) is composed of 13 core member organizations serving the licensed complementary and integrative healthcare, formerly referred to as CAM, disciplines of chiropractic medicine, acupuncture and Oriental medicine, naturopathic medicine, massage therapy, and direct-entry midwifery. In addition, ACCAHC's members include four organizations from the nonlicensed Traditional World Medicines fields of yoga therapy, Ayurvedic, and homeopathic medicine that are engaging in regulatory efforts. The ACCAHC disciplines provide the majority of the complementary and integrative healthcare for consumers in the United States. These disciplines represent over 175 U.S. Department of Education-recognized and accredited schools and programs and more than 375,000 practitioners, many of whom are first contact providers. We view the disciplines represented by ACCAHC as major stakeholders of the former National Center for Complementary and Alternative Medicine (NCCAM), recently renamed the National Center for Complementary and Integrative Health (NCCIH).
NCCAM and ACCAHC dialog, 2009-2011

In 2009, NCCAM began seeking stakeholder input on its 2011-2015 Strategic Plan. ${ }^{1}$ The ACCAHC Research Working Group (see Table 1 for members) responded in a series of communications with NCCAM officials from 2009 to 2011, including a direct meeting in 2011. In response to the question, "What will happiness mean for the public health, CAM disciplines and NCCAM in 2015?" posed by NCCAM officials, ACCAHC identified three areas as the most impactful, shared priorities for research investment across our licensed professions. These were (1) research on whole disciplines; (2) costs, cost effectiveness, cost-offsets, and cost-savings; and (3) building capacity within the disciplines' universities, colleges, and programs. These communications were approved by the ACCAHC Board of Directors before submission. $^{2}$

The NCCAM's 1998 mandate from Congress appears to be remarkably supportive of these priorities. ${ }^{3}$ We note Congress's prioritization of evaluation of outcomes and health services research. Congress calls repeatedly for the evaluation of not only "modalities" but also "disciplines" and "systems," and specifically their integration with conventional

Table 1. Members and Affiliations of ACCAHC Research Working Group

\section{Co-Chairs}

Martha Brown Menard, PhD, LMT

Cheryl Hawk, DC, PhD, CHES
Director, Crocker Institute; Research Faculty, Saybrook University; Editor, Global Advances in Health and Medicine

Associate Vice President of Research and Health Policy, Logan University; Editor in Chief, Topics in Integrative Health Care

\section{Members}

Belinda (Beau) Anderson LAc, $\mathrm{PhD}$

David Barnes, $\mathrm{PhD}^{\mathrm{a}}$

Iris Bell, $\mathrm{MD}, \mathrm{PhD}, \mathrm{MD}(\mathrm{H})^{\mathrm{a}}$

Claudia Citkovitz, MS, LAc

Ryan Bradley, ND, MPH

Jerrilyn Cambron, LMT, DC, MPH, PhD

Carlo Calabrese, ND, $\mathrm{PhD}^{\mathrm{a}}$

Gregory D. Cramer, DC, PhD

Regina Dehen, ND, LAc

Christine Goertz, DC, $\mathrm{PhD}^{\mathrm{a}}$

Mitchell Haas, DC, MA

Richard Hammerschlag, $\mathrm{PhD}^{\mathrm{a}}$

Patricia Herman, ND, MS, $\mathrm{PhD}^{\mathrm{a}}$ Janet Kahn, PhD, NCTMB ${ }^{\mathrm{a}}$

William Meeker, DC, MPH

Jeannette Painovich, DAOM, LAc, MA

Cynthia Price, PhD, LMT, NCTMB

Jennifer Rioux, PhD, CAP, RYT

James M. Whedon, DC, MS
Academic Dean, Pacific College of Oriental Medicine

Former Director of Research \& Development, Standard Process

Council on Homeopathic Education, Department of Family and Community Medicine, The University of Arizona College of Medicine Director, Acupuncture Services, Lutheran Medical Center

Assistant Director, National College of Natural Medicine Helfgott

Research Institute and School of Research and Graduate Studies

Professor, Department of Research, National University of Health Sciences Center for Natural Medicine

Professor and Dean of Research, National University of Health Sciences

Chief Medical Officer, National College of Natural Medicine

Vice Chancellor, Palmer Center for Chiropractic Policy and Research

Professor and Dean of Research, University of Western States

Past Dean of Research, Oregon College of Oriental Medicine; Past President,

Society for Acupuncture Research; Fellow, The Institute for Integrative Health Senior Behavioral Scientist, RAND Corporation

Senior Policy Advisor to the Consortium of Academic Health Centers for Integrative Medicine; former Executive Director, Integrated Healthcare Policy Consortium

President, Palmer College-West Campus

Research Acupuncturist, Cedars-Sinai Heart Institute

Research Assistant Professor, Biobehavioral Nursing and Health Systems, University of Washington

Board Member, National Ayurvedic Medical Association (NAMA); NIH/ NCCAM Research Fellow, Department of Family and Community

Medicine, University of Arizona

Dartmouth Institute for Health Policy

${ }^{\mathrm{a}}$ Past member

ACCAHC, Academic Consortium for Complementary and Alternative Health Care; NCCAM, National Center for Complementary and Alternative Medicine; NIH, National Institutes of Health. 
medicine and into delivery systems. The mandate urges evaluation of preventive approaches, and also notes the importance of investment in complementary and integrative health $(\mathrm{CIH})$ institutions as a necessary means to all of these ends. To aid in meeting this mandate, an 18-member advisory council was created. The composition of the council was specified in its charter, authorized by 42 U.S.C. 284a, section 406 of the Public Health Service Act, and states that, of the 18 , nine members should be "licensed practitioners in one or more of the major systems with which the Center is involved.",

A number of healthcare trends, beyond the mandate and the common interests and needs of the ACCAHC disciplines, supported these recommendations for NCCAM's strategic plan. Among these were an increased awareness of the multifactorial nature of care for costly chronic diseases, the heightened focus on healthcare costs, and the importance of comparative effectiveness research. ${ }^{5-8}$ In addition, the emergence of "integrative medicine" in conventional medicine $^{9}$ has prompted an overlapping interest in the evaluation of whole disciplines, whole systems, interventions related to wellness and healing, and factors related to their appropriate integration with delivery systems (Table 2). ${ }^{10-14}$ These trends are even more relevant now than in 2011.

We anticipate that such a prioritization will continue to excite increasing interest in research endeavors and continue to foster a culture of evidence among the students, faculty, and clinicians from the ACCAHC disciplines. At this time, a growing number of $\mathrm{CIH}$ researchers now have experience of the NIH culture and have participated in the NIH mission as advisors, reviewers, applicants for grants, and as funded researchers, including many of the ACCAHC Research Working Group members. We have participated in over 60 $\mathrm{NIH}$-funded projects, led review panels, and served on the
Advisory Council. Researchers and institutions associated with ACCAHC disciplines are better equipped than they were in 1998 to partner, counsel, and lead these evaluations, which Congress urges. ${ }^{15,16}$

\section{Developing $\mathrm{ClH}$ professions' research capacity}

The issue of research capacity, one of ACCAHC's top three priorities, is a focus of this commentary. ACCAHC urges the expansion of investment in the development of research infrastructure at $\mathrm{CIH}$ institutions. The career development of researchers who are graduates of $\mathrm{CIH}$ programs, particularly those who have a continued association with accredited $\mathrm{CIH}$ schools, is also necessary to accomplish the remaining two priorities. The $\mathrm{CIH}$ disciplines have gained significant new experience from programs supported by conference grants, center grants, and by the education awards that assisted the development of evidence-based medicine curriculum, research activity in our faculty and students, and partnerships with conventional academic health centers, as shown in Table 4. Graduates of a growing number of our universities, schools, and programs are benefitting from NCCIH's development awards. These have been extremely meaningful, even as NIH investment in conventional academic health centers starting a half-century ago transformed those institutions.

\section{NCCAM awards made in relation to ACCAHC research priorities}

To evaluate the congruence between ACCAHC's stated priorities and $\mathrm{NCCIH}$ funding to date, the authors examined public data from the NIH Research Portfolio Online Reporting Tool (RePORT) for fiscal years 2010-2014, the years for which actual (as opposed to estimated) funding is

Table 2. Definitions of Key Terms

Term Definition

Real-world research

Comparative effectiveness research

Whole systems research

Disciplines research
Research that seeks to capture the outcomes of usual clinical practice

"... the generation and synthesis of evidence that compares the benefits and harms of alternative methods to prevent, diagnose, treat, and monitor a clinical condition or to improve the delivery of care. The purpose of CER is to assist consumers, clinicians, purchasers, and policy makers to make informed decisions that will improve health care at both the individual and population levels." CER's distinguishing characteristics include informing a specific clinical or policy decision, comparing at least two approaches or interventions, describing results at the subgroup level, measuring benefits in real-world populations, and applying appropriate methods and data sources. ${ }^{37}$

Research that studies the complex CAM therapies and disciplines as systems-level phenomena, as opposed to single agent or having unidimensional effects, and investigates both the processes and outcomes of complex healthcare interventions or packages of care, employing research designs and strategies that are congruent with the system's explanatory model. ${ }^{38}$

"... study the integration of...disciplines with the practice of conventional medicine as a complement to such medicine and into health care delivery systems in the United States" (Section C, Public Health Service Act, 1991). Disciplines research in this context captures the outcomes of multiple members of a given discipline in such a way as to inform the decisions of third-party payers, health systems, employers, and other stakeholders for including new disciplines in healthcare delivery. A related form of disciplines research is examining practice differences, similarities, and outcomes between different disciplines or within a single discipline. It may include theories, modalities, or other therapies practiced and the rules that guide their application. ${ }^{2}$ 
Table 3. NCCAM Funding to Complementary and Integrative Health Institutions 2010-2014

\begin{tabular}{|c|c|c|c|c|c|}
\hline & \multicolumn{5}{|c|}{ Fiscal year } \\
\hline & 2010 & 2011 & 2012 & 2013 & 2014 \\
\hline $\begin{array}{l}\text { Total no. NIH grants awarded } \\
\text { for CIH research }\end{array}$ & 1623 & 1365 & 1195 & 1142 & 1100 \\
\hline Total no. NCCAM grants & 393 & 371 & 346 & 310 & 330 \\
\hline $\begin{array}{l}\text { Total no. NCCAM grants } \\
\text { awarded to CIH institutions } \\
\text { and organizations }\end{array}$ & 21 & $18(6)$ & $16(4)$ & $13(0)$ & $8(1)$ \\
\hline $\begin{array}{l}\text { Total no. NCCAM grants } \\
\text { awarded for whole systems } \\
\text { or disciplines research }\end{array}$ & $1^{\mathrm{a}}$ & $2^{\mathrm{a}}$ & $2^{\mathrm{a}}$ & 0 & $1^{\mathrm{a}}$ \\
\hline Total NIH \$ awarded for CIH & $\$ 521,416,020$ & $\$ 441,818,916$ & $\$ 493,102,989$ & $\$ 380,004,497$ & $\$ 367,150,375$ \\
\hline $\begin{array}{l}\text { Total \$ awarded from } \\
\text { NCCAM }\end{array}$ & $\$ 106,562,474$ & $\$ 107,712,655$ & $\$ 104,538,766$ & $\$ 96,676,240$ & $\$ 100,093,139$ \\
\hline $\begin{array}{l}\text { Total \$ awarded to } \mathrm{CIH} \\
\text { institutions }\end{array}$ & $\$ 6,260,157$ & $\$ 5,562,729$ & $\$ 4,726,173$ & $\$ 3,589,416$ & $\$ 2,277,643$ \\
\hline $\begin{array}{l}\text { Funding } \$ \text { awarded to } \mathrm{CIH} \\
\text { institutions as a percentage } \\
\text { of NCCAM awards }\end{array}$ & $5.87 \%$ & $5.16 \%$ & $4.52 \%$ & $3.71 \%$ & $2.27 \%$ \\
\hline $\begin{array}{l}\text { Funding } \$ \text { awarded to } \mathrm{CIH} \\
\text { institutions as a percentage } \\
\text { of NIH awards }\end{array}$ & $1.20 \%$ & $1.26 \%$ & $0.96 \%$ & $0.94 \%$ & $0.62 \%$ \\
\hline
\end{tabular}

Numbers in parentheses indicate the number of new awards.

${ }^{a}$ Non-CIH institutions.

$\mathrm{CIH}$, complementary and integrative health.

published. ${ }^{17}$ These data are presented in Tables 3-5. These numbers should be viewed in light of decreased Congressional funding authorized to NIH overall during this period. While some funding increased, particularly funding for research education at $\mathrm{CIH}$ institutions and health services research generally, relatively little funding went to licensed $\mathrm{CIH}$ clinician-researchers based at $\mathrm{CIH}$ institutions, and the percentage of NCCAM funding awarded to researchers at CIH institutions decreased from 2010 to 2014.

\section{Discussion}

We believe that one of the most significant successes of $\mathrm{NCCIH}$ in its first decade was the relatively vast expansion of research opportunities during this period for members of the licensed $\mathrm{CIH}$ disciplines, for researchers associated with the accredited $\mathrm{CIH}$ schools, and for the schools themselves. Unfortunately, these advances were not mentioned in NCCIH white papers for the 2011-2015 strategic plan. ${ }^{1} \mathrm{CIH}$ researchers have had opportunities to participate that were exceedingly rare in the past, or previously did not exist at

TABle 4. Proportions of Total NCCAM Education and Center Grants to CIH InSTITUTIONS BY YeAR AND FUNDING MECHANISM

\begin{tabular}{lccccc}
\hline & \multicolumn{5}{c}{ Fiscal year } \\
\cline { 2 - 6 } Mechanism & 2010 & 2011 & 2012 & 2013 & 2014 \\
\hline P50 (center) & $0 / 33$ & $0 / 13$ & $0 / 12$ & $0 / 12$ & $0 / 9$ \\
U19 (center) & $7 / 11$ & $3 / 4(1)$ & $4 / 4(2)$ & $2 / 5(0)$ & $0 / 17$ \\
R25 (education) & $6 / 7$ & $6 / 8(1)$ & $6 / 7(0)$ & $4 / 6(0)$ & $2 / 4(0)$ \\
\hline
\end{tabular}

Numbers in parentheses indicate the number of new awards. all. For example, chiropractic colleges have benefited from the U19 developmental center grants that established basic and clinical research support. In particular, mechanisms such the R25 education grants that encouraged research and educational partnerships between conventional and CAM institutions, researchers, clinicians, and educators are fostering a culture of inquiry and evidence-informed practice within CAM institutions. ${ }^{15,18-24}$ NCCIH's investment to date has played a significant role in creating a leadership group from the licensed CAM disciplines.

\section{General recommendations}

While progress has been made, the playing field of research funding for researchers principally associated with the licensed $\mathrm{CIH}$ disciplines and their institutions has not been, nor is it today, level, as shown in Table 3. We believe that affirmative, directive programs from NCCIH in 20162020 can significantly advance the quality and quantity of $\mathrm{CIH}$ research contributions, particularly to bettering public health. NCCAM had spoken of organizing the 2011-2015 strategic plan around a set of principles. ACCAHC identifies three principles that we believe remain central as $\mathrm{NCCIH}$ looks forward:

1. Research $\mathrm{CIH}$ the way it is practiced. This is congruent with NCCIH leadership's interest in more "real-world research."25,26 It is also congruent with the endorsement by the Consortium of Academic Health Centers for Integrative Medicine (CAHCIM) of both more "real-world effectiveness trials" and a balanced portfolio. ${ }^{27}$ High-quality $\mathrm{CIH}$ research requires appropriately trained researchers from the licensed $\mathrm{CIH}$ disciplines. Clinician-researchers from our 
Table 5. NCCAM Health Services Research Funding 2010-2014

\begin{tabular}{lccccc}
\hline & \multicolumn{4}{c}{ Fiscal year } \\
\cline { 2 - 5 } & 2010 & 2011 & 2012 & 2013 & 2014 \\
\hline Total no. NCCAM grants for HSR & 24 & 25 & 22 & 26 & 27 \\
No. HSR grants to CIH institutions & 1 & $2(1)$ & $2(1)$ & $2(0)$ & $1(1)$ \\
Total \$ NCCAM awards for HSR & $\$ 9,010,175$ & $\$ 11,614,729$ & $\$ 8,563,875$ & $\$ 8,377,013$ & $\$ 7,933,589$ \\
Total \$ to CIH institutions for HSR & $\$ 67,160$ & $\$ 780,538$ & $\$ 757,367$ & $\$ 744,411$ & $\$ 230,902$ \\
\$ to CIH institutions as a \% of total & $0.75 \%$ & $6.72 \%$ & $8.84 \%$ & $8.89 \%$ & $2.91 \%$ \\
NCCAM HSR funding & & & & \\
\hline
\end{tabular}

Numbers in parentheses indicate the number of new awards. HSR, health services research.

fields, many of whom hold PhDs, or those employed by or principally affiliated with $\mathrm{CIH}$ schools, are more likely to frame questions that authentically capture the disciplines' practices, compared to researchers more familiar with randomized controlled trials (RCTs). Continuing to invest in research infrastructure and research education at $\mathrm{CIH}$ institutions is necessary to research complementary and integrative care the way it is practiced.

2. Balance funding between basic science (bench science and efficacy trials to move science forward) and health services research (effectiveness and economics to provide information for health policy). This principle would guide NCCIH toward health services and outcomes research funding to a level that is on par with the levels of resources dedicated to basic research and that dedicated to efficacy trials. Data from RePORT presented in Table 5 show that funding for health services research from NCCAM to CIH institutions increased by a factor of 10 from 2010 to 2013; however, an increase from less than $1 \%$ to $9 \%$ is still quite small. Relatively few awards went to $\mathrm{CIH}$ institutions, and funding decreased in 2014.

3. Focus on the role of $\mathrm{CIH}$ therapies and disciplines in prevention and health promotion. It would be useful to develop reliable, valid, and comprehensive outcome measures for health and wellness that can be used in pragmatic studies. Some progress has been made already in this area. ${ }^{28,29}$ Such measures would also be useful for practice-based research networks (PBRNs) focused on questions related to prevention and health promotion, based within $\mathrm{CIH}$ institutions or in partnership with conventional academic institutions.

These principles support the outcomes, health services, and pragmatic directions of the Congressional mandate noted in the NCCAM white paper created to shape thinking on the 2011-2015 strategic plan. ${ }^{1}$ We hope to see these principles at the core of the next NCCIH strategic plan. In support of these principles, we also recommend that the NCCIH Advisory Council increase the current number of clinician-researchers from the licensed $\mathrm{CIH}$ disciplines from three $^{30}$ to nine.

\section{Specific recommendations: what would make us happy}

The R25 programs are a good bridge to the issue at the core of "disciplines research" described in Table 2. Examining the impact the ACCAHC disciplines have on the health of the public is closely linked, at every level, with the question of capacity building in these disciplines and in their educational institutions. Disciplines research is an essential consideration in addressing $\mathrm{CIH}$ institution infrastructure issues.

The value to stakeholders of NCCIH leadership in disciplines research was boosted by changes to federal law in the Patient Protection and Affordable Healthcare Act of 2010 that call for greater integration of the distinctly licensed "CAM" and integrative practice disciplines. This increased inclusion, reflecting changes already in place in many states, creates research questions throughout the payment and delivery system for which disciplines research can provide answers. Such questions might focus on the differences that adding licensed $\mathrm{CIH}$ practitioners to a patient care team makes in terms of cost, cost-avoidance or cost savings, patient experience, and health outcomes.

One potential NCCIH mechanism for engaging in disciplines research would be to create a multifaceted program that directly highlights it. Elements might include convening a focused NCCIH workshop on the topic; developing educational materials to elevate the visibility of this type of research; supporting training grants in this area; and developing a program offering that targets the outcomes relative to inclusion of disciplines that will be of use to stakeholders. As part of this multipronged effort, NCCIH could reissue variants on the traditional systems, health services, PBRNS, and observational request for applications (RFAs) that have appeared (and lapsed) in the past, with a special focus on the examinations of disciplines.

A critical mechanism for such research is the development of discipline-specific PBRNs in at least each of the core licensed disciplines of chiropractic, naturopathic medicine, acupuncture and Oriental medicine, massage therapy, and physician-led integrative medicine. PBRNs can be a key piece of community-based infrastructure for learning about how the discipline actually practices and also for disseminating quality improvement initiatives, best-practices guidelines, and for acculturating an ethic of self-evaluation in members. ${ }^{31}$ PBRNs can be effective vehicles for asking questions and generating information. PBRN initiatives are in place in chiropractic ${ }^{32}$ and discussions are underway in other fields. NCCIH's role could be to

1. support the organizing and development of disciplinespecific PBRNs;

2. offer RFAs for outcomes, quality improvement, clinical epidemiology, demonstration projects/evaluations, 
and health-promotion programs through PBRNs of licensed integrative practitioners;

3. provide training of $\mathrm{CIH}$ discipline researchers on skill sets in these research environments; and

4. support access to informatics expertise and infrastructure.

NCCIH support of such PBRNs would encourage and facilitate $\mathrm{CIH}$ institutions to invest time and resources in developing and maintaining PBRNs, which require a great deal of informational technology and data management infrastructure as well as dedicated staff. This would differ from NCCAM's prior PBRN initiative, which leaned on conventional medical PBRNs, and is distinct from the HMO network initiative. A program of $\mathrm{CIH}$ discipline-specific PBRNs could prove the most significant vehicle for generating real-world research. Partnership with academic health centers or existing PBRNs would also be useful.

\section{Research infrastructure issues}

When NCCAM initially made awards to researchers based in $\mathrm{CIH}$ institutions, few had existing infrastructure needed to support research endeavors, such as institutional review boards, administrative personnel and procedures for grants management and support, or familiarity with reporting requirements. This infusion of research funding, while welcome, made gaps in research infrastructure apparent. ${ }^{33,34}$ These disparities still exist at many $\mathrm{CIH}$ institutions, and contribute to $\mathrm{CIH}$ clinician-researchers instead seeking appointments at conventional academic institutions, or "brain drain."

Brain drain in $\mathrm{ClH}$ institutions. Present patterns of research investment and funding mechanisms often encourage brain drain. We are personally familiar with a number of researchers from $\mathrm{CIH}$ disciplines who have left $\mathrm{CIH}$ institutions to find employment in conventional academic health centers, which typically have more institutional resources available, with clearly defined career pathways. The value of collaborating with and connecting to the resources and expertise available in conventional academic health centers is helpful; however, the present pattern has two negative consequences. CIH schools are challenged to develop not only physical and administrative infrastructure but also the "intellectual infrastructure" represented by strong cadres of researchers who are onsite, employed, and working together, formulating and answering the types of questions that are of greatest significance to $\mathrm{CIH}$ fields. The opportunity to impact the educational culture of $\mathrm{CIH}$ institutions is also limited by this exportation of talent. Such trends have longterm and pervasive effects on the integration of $\mathrm{CIH}$ disciplines into mainstream healthcare.

Strategies for building sustainable $\mathrm{CIH}$ institution infrastructure. Investing in infrastructure development inside $\mathrm{CIH}$ schools and disciplines is critically important to our ongoing research contributions. We recommend the following initiatives to build research capacity in $\mathrm{CIH}$ institutions:

1. Increase the number of $\mathrm{K}$ career grants and support for early stage investigators at $\mathrm{CIH}$ institutions

2. Increase training grants awarded to $\mathrm{CIH}$ institutions (at least one per discipline)
3. Fund informatics projects, including those that support PBRNs

4. Increase awards for $\mathrm{CIH}$ clinicians or $\mathrm{CIH}$ institutionbased scientists who will focus on real-world, healthpromoting, outcomes-oriented, and health services research

5. Promote partnerships with other NIH institutes and centers in brick and mortar programs

6. Promote training for clinicians who plan to be involved in research while remaining clinicians

7. Continue and enhance the $\mathrm{R} 25$ grants for $\mathrm{CIH}$ institutions

\section{$\mathrm{NCClH}$ review processes}

Conducting $\mathrm{CIH}$ research can pose methodological challenges that often require adaptation of existing research methodologies. One issue is that $\mathrm{CIH}$ practices typically use more than one procedure/technique, and often address the whole person rather than specific symptoms. ${ }^{11,12}$ A patient of a whole-person-focused integrative practice often experiences improvement in one or more health issues during a course of treatment, even if the main complaint has not positively resolved. A second issue is that $\mathrm{CIH}$ practices often include a strong orientation toward health promotion, and not just treatment of conditions. ${ }^{1}$ These health outcomes or "positive side effects" are core values of these approaches. Additionally, practitioners often provide some degree of patient/client education and encourage self-care.

Thus, in researching $\mathrm{CIH}$, it is important to consider research methodologies such as whole systems research, ${ }^{35}$ comparative effectiveness and pragmatic trials, and qualitative research. Different types of research methodologies may need to be emphasized, such as prevention trials or longitudinal cost studies that look at overall costs rather than costs of specific conditions only. While some members of the ACCAHC research community laud insights gained from NIH reviewers, more often we hear of critiques from reviewers that seem unfamiliar with integrative health and medicine or the CAM fields. Reviewers in some cases do not appear to understand the value of clinical trial designs other than RCTs, or of qualitative research. R01 and R21 applications are often reviewed by study sections with little expertise in whole systems or in CIH. This places such proposals at a disadvantage due to lack of appreciation for the potential significance of these applications, and for innovative approaches that are more suitable for $\mathrm{CIH}$, such as determining effectiveness and other real-world research methodologies. $\mathrm{CIH}$ researchers may consequently frame research questions and methods to satisfy reviewers who are less knowledgeable about CAM, rather than in ways that may best advance science and public health. In view of these issues, ACCAHC recommends that

1. NCCIH have both basic and clinical science special emphasis panels with members knowledgeable in whole systems and disciplines research to review grants;

2. a majority of special emphasis panel reviewers have significant $\mathrm{CIH}$ knowledge and experience; and

3. NCCIH create and offer educational programs to train faculty in the licensed $\mathrm{CIH}$ disciplines and/or practitioners with research-oriented academic degrees to serve as reviewers. 


\section{Recommendations on research stage guidelines}

Since its creation, NCCAM emphasized clinical research that utilizes experimental designs such as RCTs. A welldesigned RCT is based upon established descriptive and observational research, including qualitative research. Such prior research supports the conceptualization and design of experimental studies. For many CIH disciplines, a body of prior research has often not been established. Consequently, the use of a study design like an RCT is often premature and does not reflect the underlying theoretical model or real-world practice of many $\mathrm{CIH}$ disciplines. As a result, it often has little generalizability or clinical relevance.

$\mathrm{NCCIH}$ does not have clear guidelines in place to facilitate development of a program of study related to a particular research topic or area, and the related appropriate funding mechanisms. This can lead to an expectation of higher level research questions for an exploratory (R21) mechanism, for example. We recommend that NCCIH

- publish, or adapt from other institutes, a set of principles that underscores the fundamental elements of developing a program of research, and the step-by-step processes involved in building a program of research appropriate for exploring $\mathrm{CIH}$ issues that can be used by applicants and reviewers alike, and

- develop new funding mechanisms that support fundamental questions associated with stage 1 research questions $^{36}$ that may include qualitative, descriptive, and observational studies. Additional initiatives such as the Exploratory Clinical Trials of Mind and Body Interventions for NCCIH High Priority Research Topics (R34, PAR-14-182) are encouraged.

\section{Specific programmatic recommendations}

In the last 11 years, NCCAM grants have significantly boosted the ability of many of $\mathrm{CIH}$ institutions and researchers to participate in the research endeavor. We would like to see some key existing programs continued, others expanded, and new programs created. To increase capacity of $\mathrm{CIH}$ discipline researchers, we recommend that $\mathrm{NCCIH}$

1. Continue and expand R25 grants for education in evidence-based healthcare and evidence-informed practice at $\mathrm{CIH}$ schools. Previous awards limited indirect costs to $8 \%$, and we recommend an increase in indirect costs associated with these grants to build research infrastructure.

2. Disincentivize brain drain from $\mathrm{CIH}$ institutions by supporting career development grants for clinicians from the licensed $\mathrm{CIH}$ disciplines who are affiliated with and dedicated to continuing to work in accredited CIH schools.

3. Fund additional stand-alone grants to $\mathrm{CIH}$ institutions that already have a strong research foundation, and collaborate with the NIH institutes and centers to create research infrastructure in these institutions.

4. Stimulate a higher percentage of grants to conventional centers to either require or strongly encourage partnership with $\mathrm{CIH}$ institutions or $\mathrm{CIH}$ researchers affiliated with $\mathrm{CIH}$ institutions, or give a higher priority to those that do.
5. Fund research conferences, workshops, and symposia developed through the accredited $\mathrm{CIH}$ schools, including those that explore the best methods for studying the impact of whole disciplines.

6. Following the present NIH policy of giving priority to new researchers, we urge NCCIH to give a marginal benefit to grant applications from $\mathrm{CIH}$ scientistpractitioners and applications coming from $\mathrm{CIH}$ academic/research institutions, to acknowledge that $\mathrm{CIH}$ concepts require specialized expertise to translate to conventional perspectives.

An expanded R25 program would be especially useful. These grants have been instrumental in changing the culture of recipient institutions. Through educating faculty in evidenceinformed practice, a focus on evidence has been introduced into $\mathrm{CIH}$ programs across all subdisciplines. Efficient dissemination strategies can be developed and supported by NCCIH through which previously unfunded CIH schools can partner with past R25 grant recipients. While nominally education grants, these are critically important to support other aspects of disciplines research.

\section{Summary}

The ACCAHC Research Working Group and Board of Directors commends NCCIH for its previous efforts to support high-quality research in the $\mathrm{CIH}$ disciplines. We hope that $\mathrm{NCCIH}$ will continue these efforts as it develops its 2016-2020 strategic plan. We believe that our recommendations to prioritize research based on whole disciplines, encourage the collection of outcome data related to costs, and further support capacity-building within $\mathrm{CIH}$ universities, colleges, and programs will be a strategic use of funds that can benefit the nation's health. By following these recommendations, NCCIH will support $\mathrm{CIH}$ disciplines to engage in research that will improve understanding of the way that licensed integrative health and medicine practitioners impact healthcare delivery and potentially reduce costs.

\section{Authors' Contributions}

All authors made intellectual contributions to the concepts and recommendations discussed. C.C. and J.W. articulated the concepts of "whole disciplines" and "disciplines research." M.B.M. led a workgroup that further developed these concepts, and aggregated the RePORT data on NCCAM funding and Advisory Council numbers. G.D.C. and J.W. led the meeting and correspondence with NCCAM. All authors presented to NCCAM staff and participated in the meeting. M.B.M., B.A., G.D.C., W.M., and J.W. helped draft the article. All authors read and approved the final article.

\section{Acknowledgments}

We thank Cheryl Hawk, DC, PhD, CHES, for her assistance in editing the article, and our colleagues past and present in the Research Working Group for their collective contributions to stimulating discussions of these issues. We also thank Standard Process for donating the cost of the open access publication fee. 


\section{Author Disclosure Statement}

The authors declare that they have no competing interests.

\section{References}

1. National Center for Complementary and Alternative Medicine. Draft Strategic Plan. Bethesda, MD: National Institutes of Health, 2010.

2. Weeks J. Research Working Group Communication with NIH NCCAM Feb 2011. Online document at: http:// accahc.org/images/stories/2011-15_strat_plan.pdf, accessed January 31, 2015.

3. Title VI, Section 601 of the Omnibus Appropriations Act of 1999 (P.L. 105-277). Online document at: www.gpo.gov/ fdsys/pkg/PLAW-105publ277/pdf/PLAW-105publ277.pdf, accessed January 26, 2015.

4. National Center for Complementary and Integrative Health. Membership and designation. Online document at: https:// nccih.nih.gov/about/naccih/charter\#membership, accessed January 26, 2015.

5. Kligler B, Homel P, Blank AE, et al. Randomized trial of the effect of an integrative medicine approach to the management of asthma in adults on disease-related quality of life and pulmonary function. Altern Ther Health Med 2011;17:10-15.

6. Kligler B, Homel P, Harrison LB, et al. Cost savings in inpatient oncology through an integrative medicine approach. Am J Manag Care 2011;17:779-784.

7. Tinetti ME, Basu J. Research on multiple chronic conditions: Where we are and where we need to go. Med Care 2014;52 Suppl 3:S3-S6.

8. Witt CM, Chesney M, Gliklich R, et al. Building a strategic framework for comparative effectiveness research in complementary and integrative medicine. Evid Based Complement Alternat Med 2012;2012:531096.

9. Maizes V, Rakel D, Niemiec C. Integrative medicine and patient-centered care. Explore 2009;5:277-289.

10. Fonnebo V, Grimsgaard S, Walach H, et al. Researching complementary and alternative treatments - the gatekeepers are not at home. BMC Med Res Methodol 2007;7:7.

11. Menk Otto L, Howerter A, Bell IR, Jackson N. Exploring measures of whole person wellness: Integrative well-being and psychological flourishing. Explore 2010;6:364-370.

12. Verhoef MJ, Mulkins A, Kania A, et al. Identifying the barriers to conducting outcomes research in integrative health care clinic settings - a qualitative study. BMC Health Serv Res 2010;10:14.

13. Hawk C, Ndetan H, Evans MW Jr. Potential role of complementary and alternative health care providers in chronic disease prevention and health promotion: An analysis of National Health Interview Survey data. Prev Med 2012;54: $18-22$.

14. Jonas WB, Eisenberg D, Hufford D, Crawford C. The evolution of complementary and alternative medicine (CAM) in the USA over the last 20 years. Forsch Komplementmed 2013;20:65-72.

15. Evans R, Maiers M, Delagran L, et al. Evidence informed practice as the catalyst for culture change in CAM. Explore 2012;8:68-72.

16. Connelly EN, Elmer PJ, Morris CD, Zwickey H. The Vanguard Faculty program: Research training for complementary and alternative medicine faculty. J Altern Complement Med 2010;16:1117-1123.
17. Research Portfolio Online Reporting Tools (RePORT). Funding. Online document at: http://report.nih.gov/ categorical_spending.aspx, accessed January 26, 2015.

18. Haas M, Leo M, Peterson D, et al. Outcomes from the UWS Evidence-Based Practice R25 Grant: Knowledge, attitudes, skills, and behaviors. J Manipulative Physiol Ther 2012;35:701-709.

19. LeFebvre R, Peterson D, Haas M. Evidence-based practice and chiropractic care. J Evid Based Complementary Altern Med 2012;18:75-79.

20. Taylor B, Delagran L, Baldwin L, et al. Advancing integration through evidence informed practice: Northwestern Health Sciences University's integrated educational model. Explore 2011;7:396-400.

21. Wayne PM, Hammerschlag R, German JS, Chapman T. Attitudes and interests toward research among students at two colleges of acupuncture and Oriental medicine. Explore 2010;6:22-28.

22. McCarty RL, Fenn R, Gaster B, et al. Building bridges: Qualitative assessment of a clinical faculty exchange between a naturopathic and an allopathic medical training program. Explore 2011;7:249-253.

23. Sullivan BM, Furner SE, Cramer GD. Development of a student mentored research program between a complementary and alternative medicine university and a traditional, research intensive university. Acad Med 2014;89: 1220-1226.

24. Cramer GD, Guiltinan J, Maiers M, et al. Benefits, challenges and culture change related to collaborations between complementary and alternative medicine and traditional research-intensive institutions. Medical Science Educator October 2014. Online document at: http://link.springer .com/article/10.1007\%2Fs40670-014-0077-3\#, accessed February 6, 2015.

25. National Center for Complementary and Integrative Health. NCCIH Research Blog. Online document at: https://nccih .nih.gov/research/blog/observational-secondary, accessed January 26, 2015.

26. National Center for Complementary and Integrative Health. Strategic Objective 3. Online document at: https://nccih.nih .gov/about/plans/2011/objective3.htm, accessed January 26, 2015.

27. The Integrator Blog. NCCAM Strategic Plan: Positions of CRN (supplements), AMTA (massage) and CAHCIM (MD/IM academics). Online document at: http://theintegrator blog.com/index.php?option=com_content\&task=view\&id = 615\&Itemid = 189, accessed January 26, 2015.

28. Bell IR, Caspi O, Schwartz GE, et al. Integrative medicine and systemic outcomes research: Issues in the emergence of a new model for primary health care. Arch Intern Med 2002;162:133-140.

29. Bell IR, Cunningham V, Caspi O, et al. Development and validation of a new global well-being outcomes rating scale for integrative medicine research. BMC Complement Altern Med 2004;4:1.

30. National Center for Complementary and Integrative Health. NACCIH Member Roster. Online document at: https://nccih .nih.gov/about/naccih/roster, accessed January 28, 2015.

31. Westfall JM, Mold J, Fagnan L. Practice-based research"Blue Highways" on the NIH roadmap. JAMA 2007;297: 403-406.

32. Bussieres A, Cote $\mathrm{P}$, French $\mathrm{S}$, et al. Creating a chiropractic Practice-Based Research Network (PBRN): Enhancing the 
management of musculoskeletal care. J Can Chiropr Assoc 2014;58:8-15.

33. Wayne PM, Pensack LM, Connors EM, et al. Increasing research capacity at the New England School of Acupuncture: Building grants management infrastructure. Altern Ther Health Med 2008;14:56-64.

34. Wayne PM, Buring JE, Davis RB, et al. Increasing research capacity at the New England School of Acupuncture through faculty and student research training initiatives. Altern Ther Health Med 2008;14:52-58.

35. Ritenbaugh C, Hammerschlag R, Dworkin SF, et al. Comparative effectiveness of traditional Chinese medicine and psychosocial care in the treatment of temporomandibular disorders-associated chronic facial pain. J Pain 2012; 13:1075-1089.

36. Aickin M. The importance of early phase research. J Altern Complement Med 2007;13:447-450.
37. Institute of Medicine. Initial National Priorities for Comparative Effectiveness Research. Washington, DC: The National Academies Press, 2009.

38. Ritenbaugh C, Verhoef M, Fleishman S, et al. Whole systems research: A discipline for studying complementary and alternative medicine. Altern Ther Health Med 2003;9: $32-36$.

Address correspondence to: Martha Brown Menard, PhD, LMT Crocker Institute 4551 Park Lake Drive Kiawah Island, SC 29455

E-mail: mbmenard@crockerinstitute.org 